\title{
Desarrollo y Producción de Oca (Oxalis tuberosa) e Isaño (Tropaeolum tuberosum) Bajo Dos Niveles de Fertilización
}

\author{
Grover Valdivia ${ }^{1}$, Andre Devaux ${ }^{1,2}$ Silvia Gonzáles $^{1}$, Juan
} Herbas $^{3}$, Robert J. Hijmans ${ }^{2}$

\begin{abstract}
Resumen
En la Estación Experimental Toralapa ubicada a 3,450 msnm, se estudió la influencia de la fertilización mineral sobre el desarrollo y la producción de oca e isaño con niveles de N P K de 80-160-80 kg/ha, frente a un testigo sin fertilización. En ambas especies, se observó un efecto marcado de la fertilización sobre el índice de área foliar, cobertura foliar, duración de follaje y sobre la acumulación de biomasa. Los rendimientos de los tratamientos con fertilización fueron de $23 \mathrm{t} /$ ha para la oca y $36 \mathrm{t} / \mathrm{ha}$ para el isaño, siendo de $25 \%$ (isaño) a $80 \%$ (oca) superiores a los tratamientos sin fertilización. En isaño, hubo una diferencia en la respuesta a la fertilización según las variedades. Hubo una alta correlación entre acumulación de materia seca y la radiación interceptada por el follaje. En suelos relativamente pobres, sobre todo en nitrógeno y fósforo, la fertilización mineral puede generar un incremento fuerte de los rendimientos de estos cultivos "rústicos".
\end{abstract}

Palabras claves adicionales: Fertilización, cobertura foliar, acumulación de fitomasa, Bolivia, cultivos andinos.

Aceptado para publicación: septiembre 1998.

\footnotetext{
Programa de Investigación de la Papa (PROINPA), Casilla 4285, Cochabamba, Bolivia. Centro Internacional de la Papa (CIP), Lima, Perú.

Universidad Mayor de San Simón, Cochabamba, Bolivia.
} 


\title{
Growth and Production of Oca (Oxalis tuberosa) and Isaño (Tropaeolum tuberosum) Under Two Levels of Soil Fertilization
}

\author{
Summary
}

At the Toralapa Experimental Station, located at 3,450 m.a.s.l., the effect of mineral fertilization on the development and growth of oca and isaño was studied with N P K at 80-160-80 kg/ha in comparison with a control treatment without fertilizer. In both species, the application of fertilizers had a strong effect on leaf area index, ground cover, foliage duration and on the accumulation of biomass. The yield of the treatments with fertilization reached 23t/ha for oca and $36 \mathrm{t} / \mathrm{ha}$ for isaño. This is an increase of $80 \%$ for oca and $25 \%$ for isaño, relative to the non-fertilized treatments. In isaño, the effect of mineral fertilization on yield and crop development was different for the two varieties tested. There was a strong correlation between the accumulation of dry matter and the intercepted radiation by foliage. Under relatively poor soil conditions, with low levels of nitrogen and phosphorus, mineral fertilization can contribute to high yield increase of these "rustic" crops.

Additional Index words:

Fertilization, ground cover, dry matter accumulation, Bolivia, Andean crops.

\section{Introducción}

La región Andina ha sido el centro de domesticación de muchos cultivos, entre ellos se encuentran los tuberosas Andinas: la papa (siete especies de Solanum), la oca (Oxalis tuberosa), el olluco (Ullucus tuberosas) y el isaño (Tropaeolum tuberosum) (6). Hoy en día, una especie de papa, Solanum tuberosum, es uno de los cultivos más importantes del mundo por lo que ha sido el tema de mucha investigación. Las demás tuberosas Andinas han sido poco estudiadas y valorizadas en el mundo agronómico (9). La investigación descrita en este artículo es parte del Programa

Los autores agradecen a Rolando Oros por su ayuda en la preparación del manuscrito. 
Colaborativo de Biodiversidad de Raíces y Tubérculos Andinos (2) apoyado por la Cooperación Suiza al Desarrollo (COSUDE) y es un aporte al conocimiento de estos cultivos casi olvidados por la ciencia.

En Bolivia hay datos estadísticos disponibles sobre la producción de oca mientras que para el isaño no hay, por la poca comercialización del cultivo que es utilizado para el uso interno de las fincas sobre todo para alimentar cerdos. En el caso de la oca se estima a 13,000 ha la superficie cultivada en Bolivia con la mayor concentración en el departamento de La Paz con 5,000 ha, seguido de Cochabamba con 3,800 ha (1). Las pocas investigaciones realizadas sobre los tubérculos andinos muestran que se trata de cultivos que tienen altos niveles de producción. Por ejemplo, los rendimientos en parcelas de agricultores en las principales zonas productoras de tubérculos andinos del Departamento de Cochabamba, Bolivia, alcanzan en promedio de 25 a 28 t/ha para la oca y de 30 a 48 t/ha en el isaño (4). Contrariamente, los reportes oficiales de Bolivia indican bajos rendimientos para estos tubérculos, $3.2 \mathrm{t} / \mathrm{h}$ en oca y no hay información para el isaño (1).

La producción de los cultivos depende de su potencial genético y de la interacción con el ambiente en que se desarrollan. Entre los factores ambientales, la disponibilidad de nutrientes en el suelo juega un papel determinante. Trabajos de fertilización realizados en el Perú indican que la oca responde a niveles medios de nitrógeno, muestra una alta respuesta al fósforo y al potasio. Un nivel de fertilización adecuado para las condiciones del Cusco es de 60-120-120 de N-P-K (10). Utilizando un sistema semi-hidropónico en el cual se estableció que el crecimiento y rendimiento del isaño disminuye cuando hay deficiencia de nitrógeno y fósforo, no ocurriendo lo mismo con la deficiencia de potasio (5).

Por otro lado, mediante una sondeo realizado con agricultores de las principales zonas productoras de tubérculos andinos del Departamento de Cochabamba, Bolivia, se observa que el uso de fertilizantes químicos u orgánicos de estos cultivos es bajo, aproximadamente un 60 a $70 \%$ menos de lo que normalmente se aplica al cultivo de papa. Los agricultores indican que para estos cultivos es suficiente el remanente de fertilización que deja el cultivo anterior que es papa. Los suelos de estas zonas presentan un alto contenido de materia orgánica y niveles moderados de $\mathrm{N}, \mathrm{P}$ y K (3).

La escasa información publicada sobre el efecto de la fertilización en la productividad de la oca y del isaño es contradictoria, en parte porque las condiciones en las diferentes zonas de producción no son las mismas. No existen publicaciones sobre el efecto de la fertilización en oca e isaño en los sistemas de producción de Bolivia. Con estos antecedentes, se realizó este ensayo preliminar en la Estación 
Experimental Toralapa con condiciones agroclimáticas conocidas, para estudiar la respuesta de la oca y el isaño a la fertilización mineral basándose en parámetros de desarrollo, crecimiento y rendimiento.

\section{Materiales y Métodos}

\section{Lugar}

Se estudió el efecto de la fertilización en el desarrollo y crecimiento de oca e isaño en un ensayo conducido en la Estación Experimental Toralapa, ubicada en la provincia Tiraque del Departamento de Cochabamba, Bolivia (latitud $17^{\circ} 30^{\prime} \mathrm{S}$, longitud $65^{\circ} 40^{\prime} \mathrm{O}$, altura 3,450 $\mathrm{msnm}$ ). El lugar tiene una precipitación media anual de $550 \mathrm{~mm}$, temperatura media anual de $10.9^{\circ} \mathrm{C}$, con una máxima media de $15.3^{\circ} \mathrm{C}$ y una media mínima de $1^{\circ} \mathrm{C}$ (Figura 1). El clima durante el periodo del ensayo (1994-1995) fue normal con una precipitación de $599 \mathrm{~mm}$ (Figura 2). Las condiciones de suelo de esta zona son consideradas de baja fertilidad, ácidos, baja materia orgánica y baja capacidad de intercambio catiónico como se indica en la Tabla 1 de análisis de suelos.

Tabla 1. Características de los suelos en las parcelas de ensayo.

\begin{tabular}{lcc}
\hline Análisis & Contenido & Condición \\
\hline $\mathrm{pH}$ & 5.00 & Acido \\
Materia orgánica $(\%)$ & 2.70 & Baja \\
$\mathrm{N}$ total $(\%)$ & 0.16 & Baja \\
$\mathrm{P}(\mathrm{ppm})$ & 12.00 & Media \\
$\mathrm{K}(\mathrm{meq} / 100$ g suelo) & 0.48 & Media \\
$\mathrm{CIC}(\mathrm{meq} / 100$ g suelo) & 4.40 & Baja \\
\hline
\end{tabular}




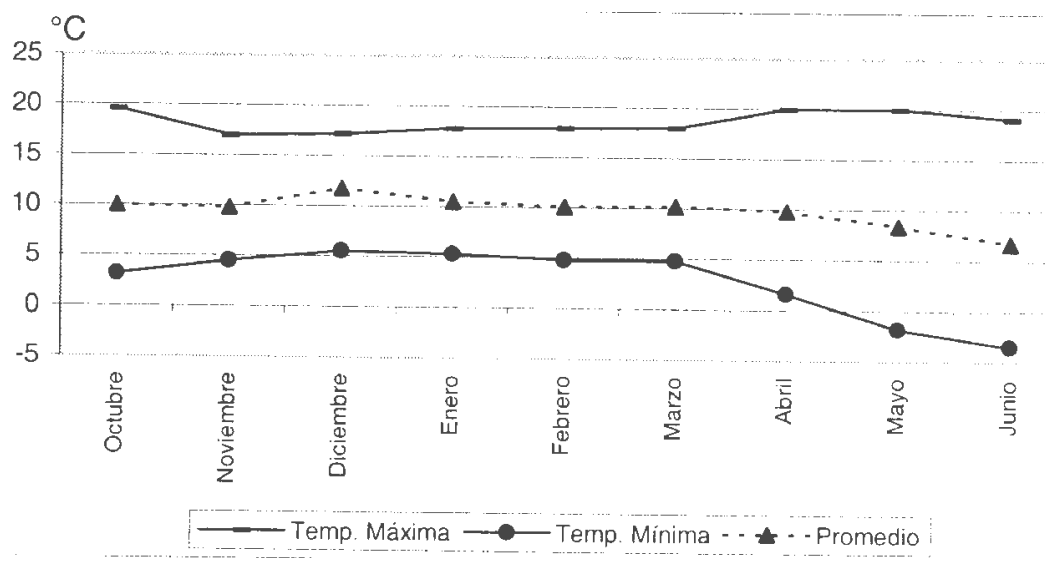

Figura 1. Temperatura máxima, mínima y promedio durante la ejecución del ensayo.

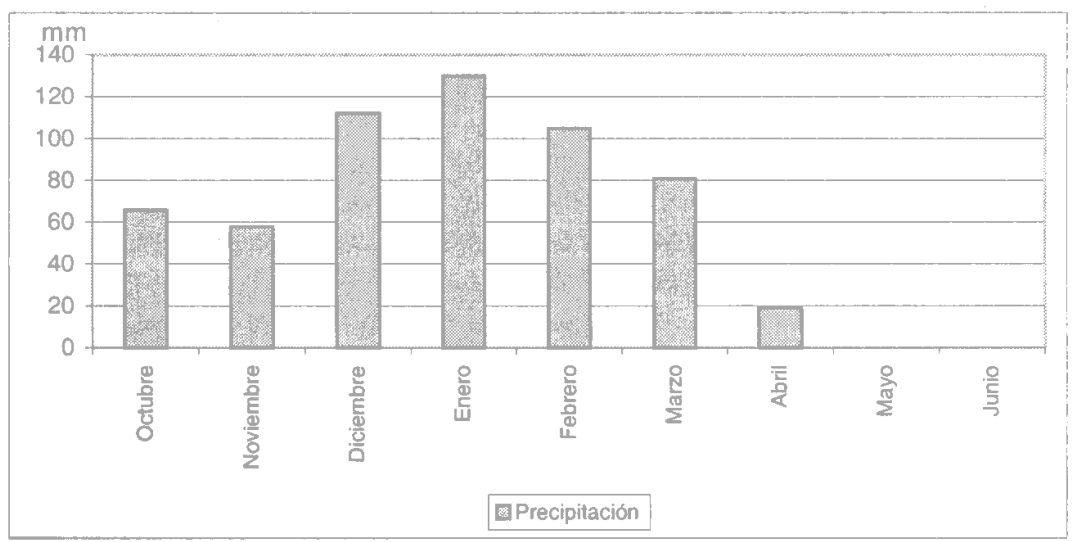

Figura 2. Precipitación promedio por mes durante la ejecución del ensayo. 


\section{Diseño y tratamientos}

Para la oca, el ensayo fue sembrado bajo un diseño de Bloques al Azar con dos tratamientos (con y sin fertilización) con cinco repeticiones. Se usó la variedad 'Puka ñawi'. La unidad experimental consistió de seis surcos de $3.6 \mathrm{~m}$ de largo con una distancia de $0.7 \mathrm{~m}$ entre surcos y $0.3 \mathrm{~m}$ entre plantas. Para el isaño se estableció el ensayo en una parcela contigua, con dos variedades: 'Anaranjada' y 'Amarilla' que fueron sembradas bajo un diseño de parcelas divididas con dos repeticiones, donde las parcelas principales fueron los tratamientos con y sin fertilización y las parcelas secundarias las dos variedades. La unidad experimental tenía 10 surcos de $4 \mathrm{~m}$ de largo, con una distancia de $0.7 \mathrm{~m}$ entre surcos y $0.4 \mathrm{~m}$ entre plantas. Las variedades utilizadas para ambos cultivos son de uso tradicional y las más difundidas entre los agricultores.

Los tratamientos con fertilizantes recibieron $80 \mathrm{~kg} / \mathrm{ha}$ de $\mathrm{N}$ en forma de urea (46\%), $160 \mathrm{~kg} / \mathrm{ha}$ de $\mathrm{P}$ en forma de superfosfato triple y 80 $\mathrm{kg} / \mathrm{ha}$ de $\mathrm{K}$ en forma de cloruro de potasio. El empleo de esta dosificación se basó en el nivel óptimo aplicado al cultivo de la papa, bajo condiciones de la Estación Experimental Toralapa, para conseguir un desarrollo adecuado de estos cultivos para los cuales se tenía poca experiencia. La aplicación del $\mathrm{P}$ y $\mathrm{K}$ se hizo a la siembra, el $\mathrm{N}$ fue fraccionado, $50 \%$ a la siembra y $50 \%$ al primer aporque.

La siembra se realizó el 28 de octubre de 1994. Las prácticas culturales durante el desarrollo del cultivo consistieron en aporques y controles fitosanitarios preventivos. El ensayo terminó con una helada de $-3.5^{\circ} \mathrm{C}$ el 10 de mayo de 1995 , a los 192 días después de la siembra (DDS).

\section{Cobertura del suelo}

Se midió la fracción del suelo cubierto por el follaje en 6 fechas $(97$, $111,121,138,161$ y $195 \mathrm{DDS})$, usando un bastidor de 0.9 por $0.7 \mathrm{~m}$, con 100 cuadros, cada uno de 7 por $9 \mathrm{~cm}$. El bastidor cubre el área de tres plantas. En cada unidad experimental se hizo tres mediciones. A través de interpolación lineal entre las observaciones, se calculó la cobertura del suelo por el follaje para cada día entre la emergencia y la senescencia. Se determinó la duración de cobertura del suelo con la siguiente formula:

$$
D C=\sum F(d)
$$


Donde:

$\mathrm{DC}=$ Duración de Cobertura [días]

$\mathrm{F}=$ Fracción del suelo cubierto por el follaje [-] en un día

$d$ = día (de emergencia hasta día de senescencia)

\section{Desarrollo}

Los parámetros de desarrollo evaluados fueron:

- Porcentaje de emergencia, observado semanalmente a partir de los 40 DDS, hasta llegar a la emergencia máxima de los cultivos.

- Inicio de la floración. Se contaron los días a partir de la emergencia hasta la aparición de los primeros brotes florales de la plantas.

- Inicio de la tuberización: Se contaron los días a partir de la siembra hasta la aparición de los primeros tubérculos. Tanto en la oca como en el isaño, se consideraron tubérculos aquellos que tenían el doble de diámetro del estolón en el que estaban insertos, siendo aproximadamente de $5 \mathrm{~mm}$ de diámetro o su materia seca superaba $1 \mathrm{~g} / \mathrm{m}^{2}(8)$.

- Inicio de la senescencia de los cultivos

\section{Crecimiento}

En cada unidad experimental, se realizaron cinco muestreos destructivos correspondientes a los 90, 102, 117135 y 197 DDS. Cada muestreo fue de cuatro plantas en oca y de seis en isaño. Las muestras fueron separadas en raíces, tallos, hojas, estolones y tubérculos, de los cuales se determinaron los pesos frescos y secos. Estos datos se utilizaron para estimar el inicio de la tuberización y para estudiar la acumulación de materia seca en el tiempo.

\section{Indice de cosecha}

Se calculó el índice de cosecha (IC) mediante la siguiente fórmula:

RA

$$
I C=\text {---------------- X } 100
$$

Donde:

RB 
$\mathrm{RA}=$ Rendimiento agronómico, el peso seco de tubérculos a la cosecha $[\mathrm{g} / \mathrm{m} 2]$.

$\mathrm{RB}=$ Rendimiento biológico, el peso seco del follaje a la madurez fisiológica más el peso seco de tubérculos a la cosecha [g/m2].

\section{Indice de área foliar}

En cada cosecha se determinó el peso (en materia seca) y el área de 20 foliólos por tratamiento. El índice de Área Foliar (IAF) fue calculado a partir de la siguiente formula:

\section{AFXPF IAF =}

\section{PS}

Donde:

$$
\begin{aligned}
& \mathrm{IAF}=\text { índice de área foliar }[-] . \\
& \mathrm{AF}=\text { Área foliar }\left[\mathrm{m}^{2}\right] \text { de los } 20 \text { foliolos } \\
& \mathrm{PF}=\text { Peso foliar }\left[\mathrm{g} / \mathrm{m}^{2}\right], \text { en material seca } \\
& \mathrm{PS}=\text { Peso seco }[\mathrm{g}] \text { de los } 20 \text { foliolos }
\end{aligned}
$$

\section{Radiación interceptada}

A través de interpolación lineal entre las observaciones, se calculó la cobertura del suelo para cada día entre la emergencia y la senescencia. Para cada día se calculó la radiación interceptada con la siguiente fórmula:

$$
\mathrm{RI}=\mathrm{F} \times \mathrm{RFA}
$$

Donde:

$$
\begin{aligned}
& \mathrm{RI}=\text { Radiación interceptada }\left[\mathrm{MJ} / \mathrm{m}^{2}\right] \\
& \mathrm{F}=\text { Fracción del suelo cubierto por el follaje }[-] \\
& \text { RFA = Radiación fotosínteticamente activa }\left[\mathrm{MJ} / \mathrm{m}^{2}\right]
\end{aligned}
$$


Sumando los valores diarios, se calculó la radiación interceptada acumulada, para las fechas de las cosechas destructivas. La radiación fue medida por un equipo automático de medición "data logger" de LI-COR (LI-1000).

\section{Resultados}

\section{Desarrollo del cultivo}

El $80 \%$ de emergencia de la oca se observó a los 50 DDS y de las dos variedades de isaño a los 54 DDS; la fertilización no afectó significativamente este parámetro. En la oca, la fertilización adelantó el inicio de la floración en 10 días y el inicio de la tuberización en 12 días (Tabla 2). Ambos cultivos crecieron hasta los 194 DDS (10 de mayo de 1995), cuando una helada de $-3.5^{\circ} \mathrm{C}$ acabó con los cultivos.

Tabla 2. Datos del desarrollo de la oca y del isaño.

\begin{tabular}{|clccccc|}
\hline Cultivo & Variedad & \multirow{2}{*}{$\begin{array}{c}\text { Emergencia } \\
\text { [DDS] }\end{array}$} & & \multicolumn{4}{c|}{ Inicio de } \\
\cline { 5 - 8 } & & & & \multicolumn{2}{c|}{$\begin{array}{c}\text { floración } \\
\text { [DDS] }\end{array}$} & \multicolumn{2}{c|}{$\begin{array}{c}\text { tuberización } \\
\text { [DDS] }\end{array}$} \\
\hline \multirow{2}{*}{ Oca Isaño } & Puka ñawi & 50 & 98 & 108 & 90 & 102 \\
& Anaranjada & 54 & 143 & 143 & 90 & 90 \\
& Amarillo & 54 & 171 & 171 & 90 & 90 \\
\hline
\end{tabular}

$\mathrm{CF}=$ con fertilizante; $\mathrm{SF}=$ sin fertilizante.

DDS = dias después de la siembra.

\section{Cobertura de suelo e índice de área foliar}

La fertilización tuvo un efecto sobre la cobertura máxima, la duración de cobertura, y el índice de área foliar en ambos cultivos y variedades. La Figura 3 muestra la expansión de la cobertura de suelo en el tiempo para ambos cultivos, donde se ve que la fertilización generó una cobertura mayor, sobre todo, en el caso de la variedad "Anaranjada" de isaño. Existen grandes diferencias en la cobertura máxima según especies, variedad y tratamiento. La oca llegó a una cobertura foliar máxima de $45 \%$ con fertilizantes y a $30 \%$ sin fertilizantes (Tabla 3). El isaño llegó a $72 \%$ y $48 \%$ con fertilizante y a $61 \%$ y $36 \%$ sin fertilizantes en las variedades "Anaranjada" y "Amarilla" respectivamente (Figura 3, Tabla 3). Ambos cultivos y tratamientos llegaron a tener una cobertura máxima alrededor de los 
110 DDS. La duración de cobertura foliar de "Anaranjada" fue superior a "Amarilla" y a la de oca. Tres días antes de la última lectura hubo una helada fuerte que afectó el follaje.
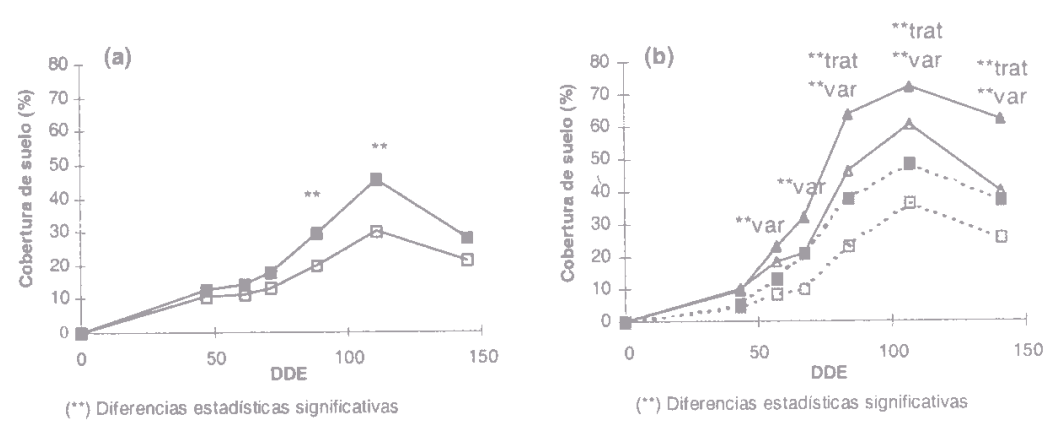

Figura 3. Cobertura de suelo en el tiempo para oca (a) e isaño (b), triángulos: Anaranjada, cuadrados: Amarilla. Símbolos cerrados: con fertilización, símbolos abiertos: sin fertilización. Diferencias estadísticas al $P=0.05$.

La oca con fertilizantes llega a un índice de área foliar (IAF) de 3.3 y 2.5 sin fertilizantes. EI IAF de 3.3 corresponde a una cobertura foliar de alrededor de $45 \%$. En papa, un IAF de 3 implica una cobertura foliar encima de $80 \%$ (11). Esto refleja el hábito monopodial y erecto de la oca. En el isaño, el IAF en "Anaranjada" fue de 5 con fertilizantes y de 4.3 sin fertilizantes; "Amarilla" alcanzó un IAF de 4.7 con fertilizantes y 3.4 sin fertilizantes. A pesar de tener un hábito mucho menos erecto que la oca, el IAF de 5 en isaño corresponde a una cobertura de suelo de sólo $72 \%$.

Tabla 3. Cobertura de suelo máxima [\%], duración de cobertura [días] e IAF máximo [-] para oca e isaño con y sin fertilización.

\begin{tabular}{|c|c|c|c|c|c|c|c|}
\hline \multirow[t]{2}{*}{ Cultivo } & \multirow[t]{2}{*}{ Variedad } & \multicolumn{2}{|c|}{$\begin{array}{c}\text { Cobertura } \\
\text { máxima }\end{array}$} & \multicolumn{2}{|c|}{$\begin{array}{c}\text { Duración de } \\
\text { cobertura }\end{array}$} & \multicolumn{2}{|c|}{ IAF máximo } \\
\hline & & $\mathrm{CF}$ & $\mathrm{SF}$ & $\mathrm{CF}$ & SF & $\mathrm{CF}$ & $\mathrm{SF}$ \\
\hline \multirow{3}{*}{$\begin{array}{l}\text { Oca } \\
\text { Isaño }\end{array}$} & Puka ñawi & 45 & 30 & 32 & 23 & 3.3 & 2.5 \\
\hline & Anaraniada & 72 & 48 & 55 & 42 & 5 & 4.3 \\
\hline & Amarilla & 61 & 36 & 35 & 24 & 4.7 & 3.4 \\
\hline
\end{tabular}

$\mathrm{CF}=$ Con Fertilizante; $\mathrm{SF}=\mathrm{Sin}$ Fertilizante 


\section{Fitomasa}

La acumulación de la fitomasa total se muestra en la Figura 4. En ambos cultivos y variedades hubo un efecto marcado de la fertilización desde el inicio del crecimiento de las plantas. La acumulación de materia seca en oca fue parecida a la de la variedad "Amarilla" de isaño. La diferencia entre las dos variedades de isaño es marcada. La materia seca acumulada de "Anaranjada" sin fertilización es mayor a la materia seca acumulada de "Amarilla" con fertilización (Figura 4, Tabla 4).
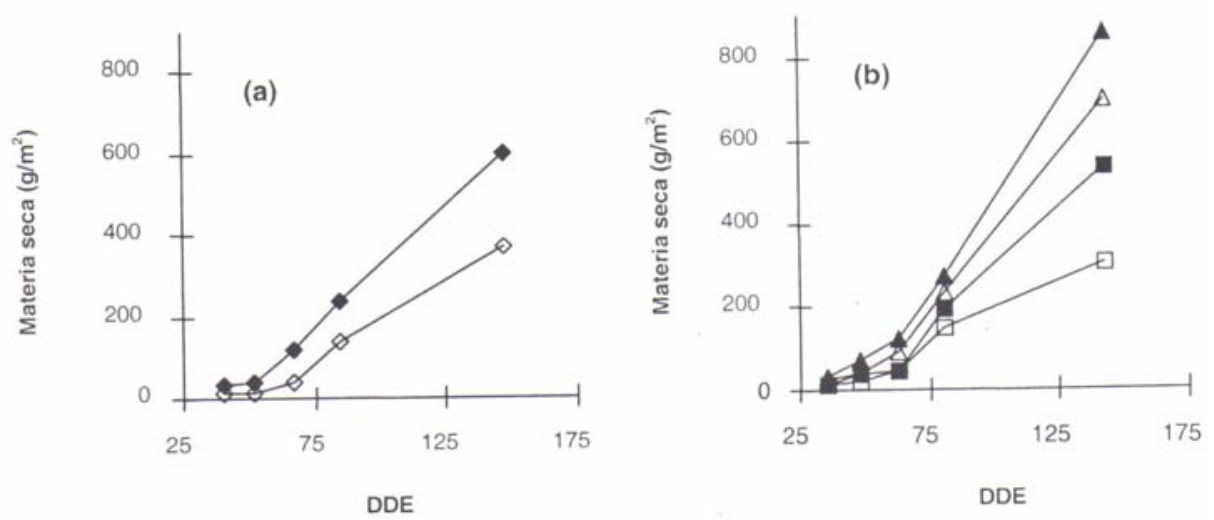

Figura 4: Fitomasa total expresada en materia seca total $\left[\mathrm{g} / \mathrm{m}^{2}\right]$ en el tiempo para la oca (a) y el isaño (b), triángulos: Anaranjada, cuadrados: Amarilla. Símbolos cerrados: con fertilización, símbolos abiertos: sin fertilización.

Tabla 4. Acumulación de materia seca (MS) total y en los tubérculos, materia fresca (MF) de los tubérculos $\left[\mathrm{g} / \mathrm{m}^{2}\right]$ e índice de cosecha (IC).

\begin{tabular}{|l|l|c|c|c|c|c|c|c|c|}
\hline Cultivo & Variedad & \multicolumn{2}{|c|}{ MS total } & \multicolumn{2}{|c|}{ MS } & \multicolumn{2}{c|}{ MF tubérculo } & \multicolumn{2}{c|}{ IC } \\
& & & & \multicolumn{2}{c|}{ tubérculo } & \multicolumn{2}{c|}{} \\
\cline { 3 - 10 } & & CF & SF & CF & SF & CF & SF & CF & SF \\
\hline Oca & Puka ñawi & $601^{*}$ & $367^{*}$ & $393^{*}$ & $234^{*}$ & $2286^{*}$ & $1271^{*}$ & 0.65 & 0.64 \\
Isaño & Anaranjada & $852^{*}$ & $696^{*}$ & $586^{* *}$ & $474^{* *}$ & $3612^{* *}$ & $2884^{* *}$ & 0.69 & 0.68 \\
& Amarilla & $531^{*}$ & $301^{*}$ & $307^{* *}$ & $157^{* *}$ & $3000^{* *}$ & $1800^{* *}$ & 0.58 & 0.52 \\
\hline
\end{tabular}

$\mathrm{CF}=$ con fertilizante; $\mathrm{SF}=$ sin fertilizante.

* significativas $(P=0.05)$ entre tratamientos.

** significativas $(P=0.05)$ entre variedades y tratamientos. 
Las diferencias en acumulación de materia seca (Figura 2) ya mostraron el efecto de la aplicación de fertilizantes sobre los rendimientos agronómicos. La oca con fertilizantes tuvo un rendimiento de 22.9 t/ha y de $12.7 \mathrm{t} / \mathrm{ha}$ sin fertilizantes. En el caso del isaño se registraron rendimientos de 36.1 t/ha con fertilizantes y de $28.8 \mathrm{t} / \mathrm{ha}$ sin fertilizantes para la variedad 'Anaranjada' y 30 y $18 \mathrm{t} / \mathrm{ha}$ para 'Amarilla'. Los rendimientos con fertilización son comparables a los reportados por Quispe et al. (8), obtenidos con un abono de estiércol de gallina a una dosis de 5 t/ha (7).

De la Tabla 4 se deduce los porcentajes de materia seca de los tubérculos en los dos cultivos de estudio. En la oca, el contenido de materia seca fue de $17.2 \%$ con fertilización y de $18.4 \%$ sin fertilización. En el isaño, en la variedad 'Anaranjada' la materia seca fue de $16 \%$ y en la variedad 'Amarilla' la materia seca fue de $10 \%$. No se observó un efecto marcado de la fertilización sobre el porcentaje de materia seca en tubérculos, pero la diferencia entre la variedades de isaño muestra la influencia de la variabilidad genética sobre este factor. La variedad 'Amarilla' tiene un índice de cosecha más bajo que 'Anaranjada' y que el cultivo de oca. Esta diferencia varietal indica la necesidad de evaluar el material genético disponible de isaño y oca para su potencial productivo.

\section{Radiación interceptada y acumulación de materia seca}

La acumulación de materia seca tuvo una correlación alta con la radiación interceptada (Figura 5). Esta relación no varió mucho entre cultivos, variedades y tratamientos. Hubo una eficiencia de uso de radiación promedio de $1.6 \mathrm{~g} / \mathrm{MJ}$ que es baja en relación a valores observados para el cultivo de papa que son alrededor de 2 a $3 \mathrm{~g} / \mathrm{MJ}$ (12). Considerando el índice de cosecha más bajo de la variedad 'Amarilla' de isaño, ésta presentó la eficiencia más baja de acumulación de materia seca en tubérculos. 


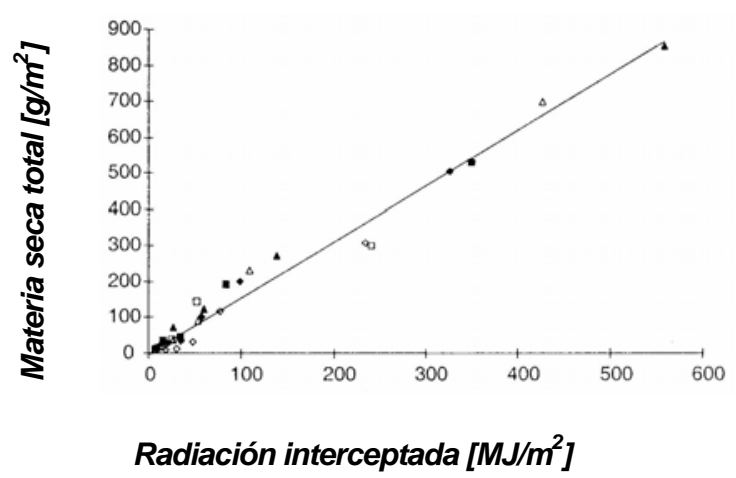

Figura 5. Acumulación de materia seca en relación con la radiación interceptada acumulada $\left(y=1.55 x, R^{2}=0.98\right)$. Los puntos representan las observaciones en los días de cosechas destructivas, oca (diamantes), isaño, 'Anaranjada' (triángulos) y 'Amarilla' (cuadrados) Símbolos cerrados: con fertilización, símbolos abiertos: sin fertilización.

\section{Conclusiones}

El efecto de la fertilización mineral sobre el desarrollo de los cultivos de oca e isaño fue similar y limitado, sólo adelantó un poco el inicio de la tuberización en oca. En cambio, el efecto sobre el crecimiento fue marcado. En la oca y el isaño, la fertilización mineral generó una mayor cobertura del follaje. Los valores máximos de IAF y la cobertura del suelo fueron más altos con fertilización además de incrementar los valores de la duración de cobertura del suelo.

Existió un efecto positivo de la fertilización sobre la acumulación de materia seca y sobre el rendimiento agronómico. La fertilización incrementó el rendimiento de oca en un $80 \%$. En el isaño, el efecto de la fertilización sobre los rendimientos no fue tan marcado como en la oca, con un incremento de $25 \%$ y $63 \%$ en la 'Anaranjada' y la 'Amarilla' respectivamente. Parece que estos cultivos "rústicos" pueden aprovechar mejor de un manejo intensificado. 
Bajo las condiciones agroecológicas de la Estación Experimental Toralapa (suelos pobres, temperaturas promedias bajas, precipitaciones irregulares), los resultados del efecto de la fertilización sobre los cultivos estudiados fueron evidentes. En cambio, las zonas productoras típicas de estos tubérculos andinos suelen tener suelos con alto contenido de materia orgánica y niveles moderados de $\mathrm{N}, \mathrm{P}$ y $\mathrm{K}$ con una pluviometría más elevada. En ensayos preliminares en estas zonas, se constató que la respuesta a la fertilización no es tan marcada como en Toralapa.

El incremento de la cobertura foliar debido a la fertilización explica las diferencias de rendimiento entre tratamientos. La radiación interceptada tuvo una correlación alta con la producción de fitomasa total. No hubo grandes diferencias entre cultivos y variedades usadas en la eficiencia global de uso de la radiación pero sí se observó una diferencia de índice de cosecha entre las variedades de isaño que implicaría una eficiencia menor de acumulación de materia seca en tubérculos para una de las dos variedades. Además en este cultivo, se notó una diferencia grande entre variedades en el desarrollo de follaje y en el rendimiento. Al parecer se trata de dos variedades muy distintas en su adaptación al lugar de ensayo o en su potencial genético. Un mejor conocimiento de las diferencias en el potencial productivo entre variedades de oca e isaño podría ayudar en aprovechar mejor la productividad de estos cultivos.

Por los altos índices de área foliar y los valores relativamente bajos de cobertura del suelo, parece que ambos cultivos podrían tener un rendimiento más alto adaptando la densidad de siembra con una menor distancia entre surcos. En el ensayo, las distancias entre surcos y plantas fueron adaptadas de las densidades usadas en el cultivo de papa y a las prácticas de los agricultores. Ensayos de densidades de siembra con diferentes variedades podrían ser útiles para mejorar el manejo agronómico.

\section{Referencias Bibliográficas}

1. Centro de Información para el Desarrollo (ed.). 1994. Anuario estadístico del sector rural, 1994. G-DRU; IICA; GTZ; COTESU; Embajada de Holanda; ILDIS; CEARS; AGRODATA. p.138-139; 153-154. La Paz, Bolivia.

2. CONDESAN-CIP. 1997. Andean root and tuber crops: a report on collaborative research in biodiversity, 1993-1997. CONDESANCIP, 27p. 
3. González, S., Devaux, A. 1994. Factores limitantes de producción de tubérculos andinos. En Informe Anual 1993-94. Programa de Investigación de la Papa (Bol). IBTA/CIP/COSUDE. p.IV 27-IV 32 y p.IX 18-1X49.

4. González, S.; Almanza, J.; Devaux, A.; Condori, P. 1997. Zonas productoras de tubérculos andinos, identificación e investigación de factores limitantes de producción y conservación en Cochabamba. En: IX Congreso Internacional de Cultivos Andinos. p.21.

5. Gómez, D.; Rodríguez, G.; Rodríguez, A. 1997. Efectos de la nutrición mineral sobre el crecimiento y rendimiento de la mahua (Tropaeolum tuberosum). En: IX Congreso Internacional de Cultivos Andinos, p.38-39.

6. National Research Council. 1989. Lost crops of the Incas: Littleknown plants of the Andes with promise for worldwide cultivation. National Academy Press, Washington, D.C. 415p.

7. Quispe, C. 1997. Parámetros agrofisiológicos del desarrollo y crecimiento de los cultivos: papa (Solanum tuberosum), oca (Oxalis tuberosa), e isaño (Tropaeolum tuberosum R.), en Toralapa, Cochabamba. Tesis de grado. Universidad Mayor de San Andrés, Facultad de Agronomía, La Paz. 110p.

8. Quispe, C.; Devaux, A.; González, S.; Tourneux, C.; Hijmans, R. 1997. Evaluación comparativa del desarrollo y crecimiento de papa, oca e isaño en Cochabamba, Bolivia. Revista Latinoamericana de la Papa 9/10(1): 140-155.

9. Tapia, M. 1997. Cultivos Andinos subexplotados y su aporte a la alimentación. 2da. Edición. Santiago, Chile, p.93.

10. Tapia, M. 1995. Avances de las investigaciones sobre tubérculos alimenticios de los Andes. Proyecto INIAA-CIID-ACDI (PISA). Lima, Perú. 113 p.

11. Tourneux, C.; Camacho, M.R.; Mamani, P.; Devaux, A. 1998. Effect of water shortage on six potato genotypes I- Morphological parameters, growth variable and yield components. Programa de Investigación de la Papa, PROINPA. Cochabamba, Bolivia. 17p.

12. Van Keulen, H. y Stol, W. 1995. Agro-ecological zonation for potato production. En: Haverkort, A.J. and D. K. L. MacKerron (eds.). Potato Ecology and Modeling of Crops under Conditions Limiting Growth. Kluwer, Dordrecht. pp. 357-379. 